\title{
Automated detection of cardiac phase from intracoronary ultrasound image sequences
}

\author{
Zheng Sun ${ }^{1 *}$, Yi Dong and Mengchan Li \\ Department of Electronic and Communication Engineering, North China Electric Power University, \\ Baoding, China
}

\begin{abstract}
Intracoronary ultrasound (ICUS) is a widely used interventional imaging modality in clinical diagnosis and treatment of cardiac vessel diseases. Due to cyclic cardiac motion and pulsatile blood flow within the lumen, there exist changes of coronary arterial dimensions and relative motion between the imaging catheter and the lumen during continuous pullback of the catheter. The action subsequently causes cyclic changes to the image intensity of the acquired image sequence. Information on cardiac phases is implied in a non-gated ICUS image sequence. A 1-D phase signal reflecting cardiac cycles was extracted according to cyclical changes in local gray-levels in ICUS images. The local extrema of the signal were then detected to retrieve cardiac phases and to retrospectively gate the image sequence. Results of clinically acquired in vivo image data showed that the average inter-frame dissimilarity of lower than 0.1 was achievable with our technique. In terms of computational efficiency and complexity, the proposed method was shown to be competitive when compared with the current methods. The average frame processing time was lower than $30 \mathrm{~ms}$. We effectively reduced the effect of image noises, useless textures, and non-vessel region on the phase signal detection by discarding signal components caused by non-cardiac factors.
\end{abstract}

Keywords: Coronary arterial vessel, intracoronary ultrasound (ICUS), motion artifact, cardiac phase, retrospective gating

\section{Introduction}

Intracoronary ultrasound (ICUS), an invasive imaging modality performed during coronary angiography, has been widely used in clinical diagnosis and interventional treatment of coronary artery diseases. During continuous pullback of the catheter, the ultrasonic probe is constantly moving with heartbeats. Both axial and lateral catheter motions subsequently cause cyclic changes to the image content (known as motion artifact), thereby showing that information on cardiac cycles and phases is implied in the continuous pullback ICUS image sequence [1-3].

In past decades, retrieval of implied cardiac phases from a non-gated ICUS image sequence based only on image information, i.e., off-line image-based gating, has attracted an increasing amount of attention from cardiac researchers. Present methods can be classified into two categories:

(1) Explore temporal changes of vascular morphometry or lumen size.

For example, in [4], the changing vessel lumen contours are segmented and tracked along the sequence. In [5], the lumen sizes are tracked according to the changes in image intensities within a

\footnotetext{
* Address for correspondence: Zheng Sun, P.O. Box 21, North China Electric Power University, Hebei, Baoding, 071003, P.R. China. Tel.: +86 15930929261; Fax: +86 03127522272; E-mail:sunzheng_tju@163.com.
} 
manually defined circular region. The rigid motion of the vessel wall in successive frames are estimated in [6] by tracking an elliptical vessel approximation. In [7], a cardiac phase is assigned to each image by tracking sharp changes in the vessel morphology. The main limitation of the aforementioned methods is that accurate segmentation of vessel wall contours in each cross-sectional frame is necessary. The accuracy of the retrieved cardiac phases is sensitive to image segmentation. In [3], the heartbeat phase is directly extracted from an ICUS video through feature tracking. Although complete segmentation is not required, this method may remain computationally expensive.

(2) Analyze changes in image intensity between successive frames.

A proper similarity or dissimilarity measurement scheme that measures cyclic changes between different frames is necessary. Most existing methods use a global similarity measure that considers all image pixels. For example, average intensity, average intensity difference, correlation coefficient, and mutual information are adopted in [8]. However, detailed validation experiments with in vivo data are not provided. An intelligate gating method is presented in [9], where the dissimilarity between frames is measured using the normalized cross-correlation (NCC) of the average intensities. A dissimilarity matrix based on the NCC between pairs of frames is constructed in [10]. The methods presented in [11] and [12] are the improved versions of the one in [10]. A motion blur intensity estimator is proposed in [13], and the estimate is computed as minus the average of the absolute value of the vertical derivative over the polar image. These global similarity measures result in the sensitivity of cardiac phase detection to background noises, useless textures, and non-cardiac dynamic phenomena. The evolution of tissue density of mass is explored in [14]. The validation results show that a local approach can effectively reduce the impact of texture and motionless regions.

Further, the phase of a moving organ system is detected in [15] from dynamic images by detecting the phase shift of the different frequencies in an image. However, this method is limited to moving objects that exhibit only translations. Manifold learning is also used to retrospectively sample ICUS sequences [16] or ultrasound and MR images [17].

This study presents a novel methodology for detecting a 1-D time-independent phase signal that correlates with the cardiac cycles according to the dynamic information embedded in the ICUS shortaxis image sequence. For each image pixel, a 1-D intensity variation signal is firstly extracted by counting its gray levels along the overall sequence. The signals properly implying cardiac motion are selected. The averaged signal is finally filtered to remove non-cardiac components, and a 1-D phase signal is obtained. The effect of non-cardiac factors on the retrieval of cardiac phases and retrospective gating is effectively reduced.

\section{Method}

\subsection{Detecting a phase signal}

For each pixel location in a short-axis image, its gray-levels along the overall sequence are counted, thereby obtaining a 1-D intensity variation signal (IVS), $g_{x, y}(m)$, where $m=1,2, \ldots, M$ is the frame index, $M$ is the total number of frames, $g \in[0,255]$ is the gray level, $x, y=0,1, \ldots, N-1$ indices are the horizontal and vertical coordinate of a pixel, and $N$ is the image height (or width) in pixels. Such signals do not contain the pixel position information. A 3-D matrix representing the image sequence is projected onto a 2-D matrix, $\boldsymbol{P}$. The value of each element, $p_{i, j}$, represents the gray level, where $i$ and $j$ denote the pixel order and the frame index, respectively. Regarding an ICUS image sequence containing $M$ frames, the dimensionality of $\boldsymbol{P}$ is $N^{2} M$. 
The intensities of the pixels within the catheter region and background region, i.e., non-vessel region outside the ring in Figure 1 basically remain unchanged along the dynamic pullback sequence. The IVSs obtained from these pixels are nearly direct current signals, which are called motionless signals. As shown in Figure 2(a), the Fourier amplitude spectra have no clear or sharp peak at the average heart rate. The spectra also do not contribute to the extraction of a 1-D phase signal.

The left signals are divided into two categories. As shown in Figure 2(b), the peak at the heart rate is not prominent compared with other harmonics because of the irregular changes in the image intensity. These signals are called interfered signals. The signals properly reflecting cardiac motion are called dynamic signals, as shown in Figure 2(c). The Fourier amplitude spectra have a prominently sharp peak at the heart rate, while the amplitudes of other frequencies are much smaller than the main peak.

Obviously, dynamic signals are useful for detecting a 1-D phase signal, and they should be selected from all IVSs. The Fourier spectrum of an IVS, $g_{x, y}(m)$, is denoted as $G_{x, y}(f)$, where $f$ is the frequency in Hz. The ratio is determined by the following formula:

$$
r_{x, y}=\left|G_{x, y}(R)\right| /\left|G_{x, y}(f)_{\max }\right|,
$$

where $\left|G_{x, y}(R)\right|$ is the amplitude of $G_{x, y}(f)$ at the average heart rate, $R$, in beats per minute (bpm), and $\left|G_{x, y}(f)\right|_{\max }$ is the peak amplitude of $G_{x, y}(f)$ except the peak at $R$. If $r_{x, y}$ is equal to or higher than a predefined threshold, $\varepsilon, g_{x, y}(m)$ is denoted as a dynamic signal and reserved; otherwise, it is discarded. The selected dynamic signals are finally averaged, obtaining a 1-D signal, $g(m)$, where $m=1,2, \ldots, M$ is the frame index and $g \in[0,255]$ is the gray level.

$R$ is obtained from the ECG signals recorded during image acquisition. Regarding those ICUS records for which associated ECG signals are not available, $R$ is estimated according to the average dissimilarity of gray levels, as detailed in [10]. If the frame rate is $F_{r}$, in fps, the approximate length of a cardiac cycle in frames is $D_{0}=60 F_{r} / R$.

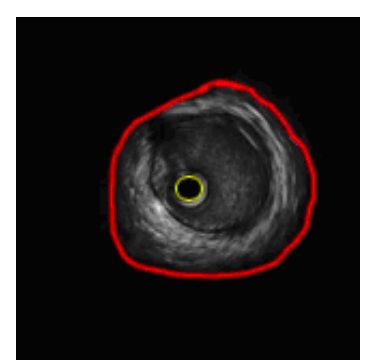

Fig. 1. An ICUS frame.

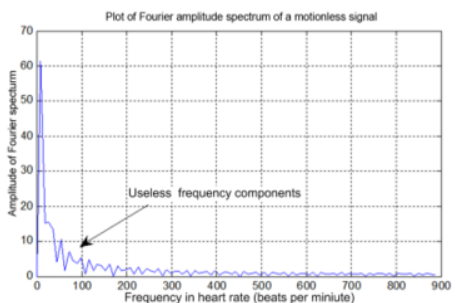

(a)

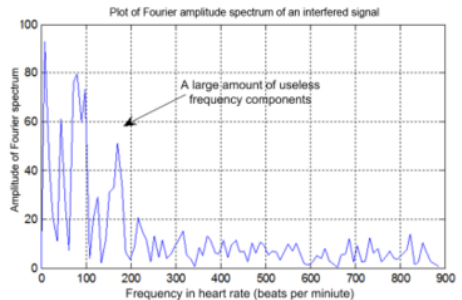

(b)

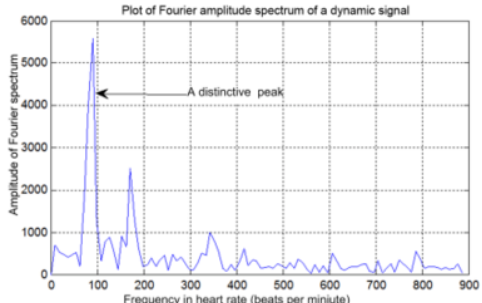

(c)

Fig. 2. Fourier amplitude spectra of a (a) motionless pixel, (b) interfered pixel, and (c) dynamic pixel. 


\subsection{Signal filtering}

The quasi-periodic signal, $g(m)$, is mainly caused by vascular morphology and cardiac motion [4, 18]. The image intensity variation caused by cardiac motion is much more rapid than the irregular vascular configuration because of the high frame rate and low pullback speed (typically at 30 fps and $0.5 \mathrm{~mm} / \mathrm{s}$ ) of the ICUS imaging. Ideally, i.e., the subject's heart rate remains constant and the image noises and computation errors are not considered, $g(m)$ should be regularly periodic, and its cycle should be equal to the subject's cardiac cycle length in frames. However, the heart rate may fluctuate during the catheter pullback, especially when the target vessel is relatively longer. Unsteady heart rate, breathing, and irregular lumen morphology may introduce irregularities in $g(m)$ and spread frequencies around the average heart rate. Consequently, a filter used to filter out frequencies not correlated with cardiac motion is crucial $[5,13,14]$.

In the present study, a band-pass filter centered on $R$ is designed to discard frequencies caused by irregularities in heartbeat and non-cardiac dynamics factors in $g(m)$. The band-pass width is set as $[R-$ $0.3 R, R+0.3 R]$ by considering heart rate variability $[5,11] . g(m)$ has the following characteristics: (1) main frequencies are distributed over a certain range; (2) the requirement of the frequency precision is not strict; (3) the requirement of the phase accuracy is very strict since the signal should reflect cardiac phases; and (4) real-time process is not necessary and quasi- or non-real-time process is acceptable. Therefore, a finite impulse response (FIR) filter is chosen, and a fast convolution structure is used to improve the efficiency. Related discussions on filter realization, order and specifications, as well as quantitative evaluation, are provided in Section 3.

The filtered signal is represented as $g^{(f)}(m)$. The local minima of $g^{(f)}(m)$ corresponding to enddiastole are finally detected, and these are taken as the sampling points to select gating frames.

\section{Validation and results}

\subsection{In vivo results}

Our method has been validated on in vivo data of 31 pullback datasets acquired from 25 patients (17 males and 8 females). Images were acquired using a Jomed Endosonic (Beringen, Switzerland) imaging system. The device is equipped with a $2.9 \mathrm{~F} 30 \mathrm{MHz}$ mechanically driven catheter, which is pulled back at a constant speed of $0.5 \mathrm{~mm} / \mathrm{s}$ driven by a motor. A series of tomographic images of the lumen is acquired at $30 \mathrm{fps}$. The image size is $360 \times 360$ pixels, and the gray level range is $[0,255]$.

The approximate average heart rate of an ICUS sequence containing 200 frames was estimated as $R$ $=90.45(\mathrm{bpm})$. The $360 \times 360$ IVSs obtained from the first 150 frames and their averaged signal are shown in Figures 3 and 4, respectively. Obviously, there is a large amount of non-cardiac frequencies in the averaged signal. A total of 71 dynamic signals with $r_{x, y}$ equal to or higher than $\varepsilon=3$ were selected, and their pixel locations were denoted as yellow crosses in an ICUS image (see Figure 5). Figure 6 shows that the periodicity of the averaged dynamic signal is much clearer than that in Figure 4. These results show that the selection of dynamic signals is effective in removing motionless and interfered pixels. A $10^{\text {th }}$ order band-pass FIR filter was designed. The central, bottom, and top stop frequency of the band-pass were set as 90.45, 63, and $118 \mathrm{~Hz}$, respectively. As shown in Figure 7, the time-domain waveform of the filtered signal is obviously smoother than the original one in Figure 6. The amplitude of the second harmonic of the heart rate was reduced to about a tenth of the main peak. 
The detected phase signal plotted with the ECG QRS trigger signal is illustrated in Figure 8, which shows that the cardiac phases are in overall agreement.

Frames corresponding to the local minima of the phase signal are selected to form a gated sequence. Two examples of conventional ICUS sequences without branching or stents are shown in Figure 9. Results of ICUS data involving vessel branching or stents are shown in Figure 10, where the gated

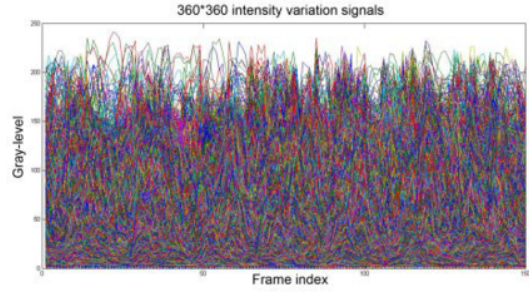

Fig. 3. $360 \times 360$ IVSs.

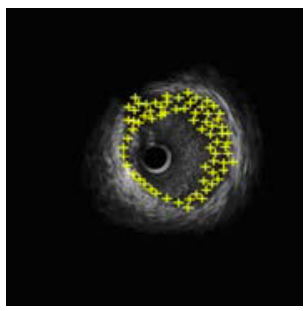

Fig. 5. An ICUS frame.
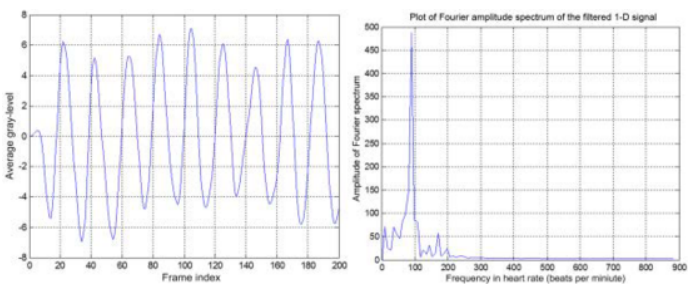

Fig. 7. Time-domain plot and Fourier amplitude spectrum of the filtered signal.
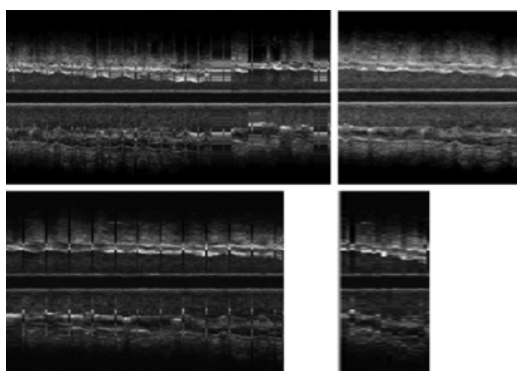

Fig. 9. Original (left) and gated (right) longitudinal cuts of two conventional ICUS sequence.
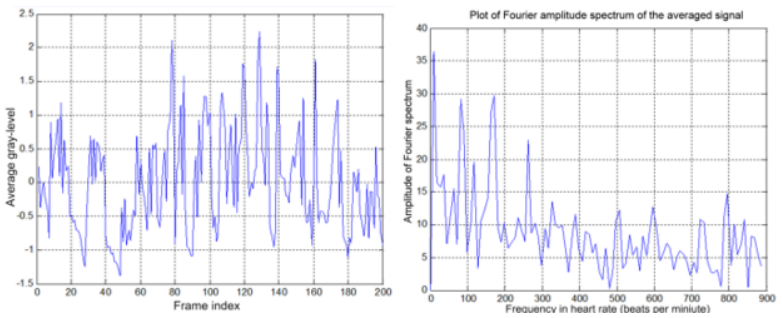

Fig. 4. Time-domain plot and Fourier amplitude spectrum of the averaged signal.
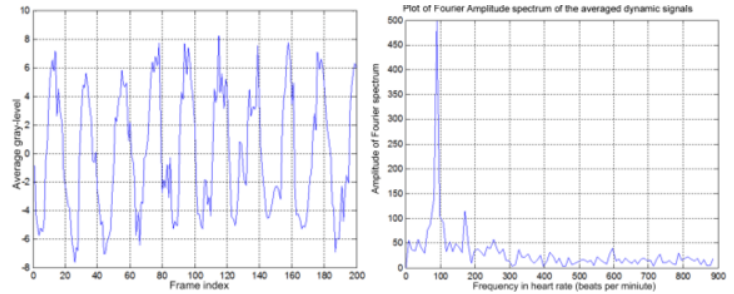

Fig. 6. Time-domain plot and Fourier amplitude spectrum of the averaged dynamic signals.

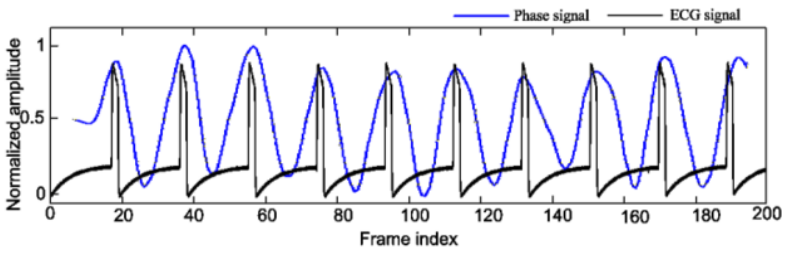

Fig. 8. Normalized phase signal and ECG signal.

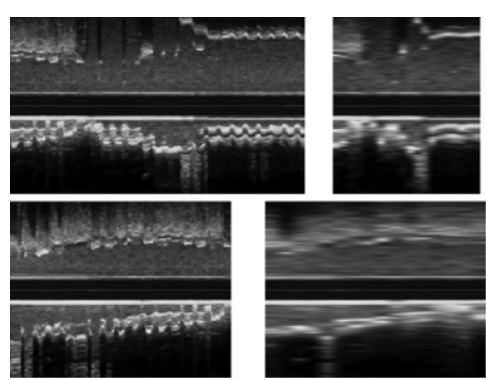

Fig. 10. Original (left) and gated (right) ICUS longitudinal cuts containing bifurcation (top) and stent (bottom). 
sequence of the bottom example is stretched to the same length as that of the non-gated one. Obviously, the serrated vessel wall borders are smoothened in the gated longitudinal cuts and thus the visual effect is improved.

\subsection{Quantitative evaluation}

The average inter-frame dissimilarity of the gated sequence, $d_{g}$, and gated frame number difference, $\Delta n=\left|n_{t}-n_{a}\right|$, were used as quantitative scores [10]. $n_{a}$ is the number of selected gating frames, and $n_{t}=n / D_{0}$ is the theoretical number of gating frames. $n$ is the total number of frames, and $D_{0}$ is the approximate cardiac cycle length in frame. Smaller $d_{g}$ and $\Delta n$ result in better the gating algorithm.

The performances of Butterworth, Chebyshev I and II IIR filter, as well as FIR filter designed with Hamming, Hanning, Blackman, Rectangular, Triangular, and Gaussian window on two ICUS sequences, were evaluated separately. The filter specifications are listed in Table 1 , where $f_{s}$ is the sampling frequency, $\sigma$ is the scale of Gaussian window, $R_{p}$ is the maximal decay of the passband, $A_{s}$ is the minimal decay of the stopband, $f_{0}, f_{1}$, and $f_{2}$ are, respectively, central, bottom stop, and top stop frequencies of the passband. Statistical results of quantitative scores are shown in Figure 11, where "(1)" and "(2)" denote the proposed method and the one in [11]. "(10)" and "(32)" denote the filter order. Obviously, the overall performances of FIR are better than those of IIR. The outputs of the FIR filter designed with different windows with the same order do not differ greatly. The performance of an FIR filter does not improve with the increase of its order. A higher order filter may have sharper cut-off characteristics. The subsequent retrospective gating may be closer to equidistant sampling. However, a regular signal may not reflect the actual heart rate variability.

The accuracy of the proposed method was compared with the method in [11] and ECG-gating. A $10^{\text {th }}$ order FIR filter designed with a Hamming window was used in the comparison experiments. Table 2 and Figure 12 illustrate the results with five image sequences, where "(1)" and "(2)" denote the proposed method and the one in [11]. In the table, $t$ is the processing time per frame in ms, which is given for a PC equipped with a $2.7 \mathrm{GHz}$ Intel Core I5-5200U CPU with 4G RAM. The processing time taken in necessary initializations (including reading in original images and outputting gating results) was not counted. From Figure 12, it is obvious that $d_{g}$ of the gated sequences are smaller than the original ones. The quantitative scores of the proposed method are better than those in [11].

Table 1

The filter specifications

\begin{tabular}{llllllll}
\hline $\begin{array}{l}\text { Seq. } \\
\text { no. }\end{array}$ & $f_{s}$ & $\sigma$ & $R_{p}$ & $A_{s}$ & $f_{0}$ & $f_{1}$ & $\boldsymbol{f}_{\mathbf{2}}$ \\
& $(H z)$ & & $(d B)$ & $(d B)$ & $(H z)$ & $(H z)$ & $(\mathbf{H z})$ \\
\hline 1 & 1800 & 2.5 & 1 & 60 & 90.45 & 63 & 118 \\
2 & & & & & 86.12 & 60 & 112 \\
\hline
\end{tabular}

Table 2

Quantitative scores of the comparison experiments

\begin{tabular}{llllll}
\hline Seq. no. & $n$ & $\Delta n^{(1)}$ & $\Delta n^{(2)}$ & $t^{(1)}(\mathrm{ms})$ & $t^{(2)}(\mathrm{ms})$ \\
\hline 1 & 1836 & 1 & 1 & 25.3 & 41.6 \\
2 & 1704 & 0 & 1 & 24.4 & 42.3 \\
3 & 2124 & 1 & 2 & 23.7 & 42.6 \\
4 & 1944 & 1 & 1 & 25.1 & 44.1 \\
5 & 2376 & 1 & 3 & 23.2 & 41.9 \\
\hline
\end{tabular}




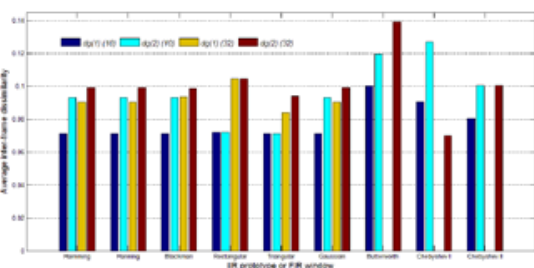

(a)

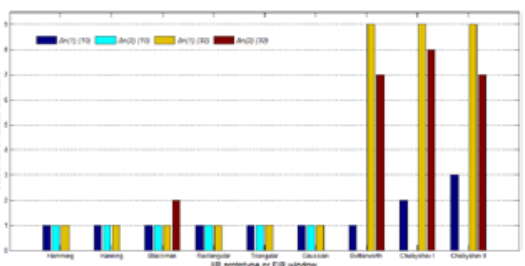

(b)

Fig. 11. Comparison of (a) $d_{g}$ and (b) $\Delta n$, with different filter prototypes.

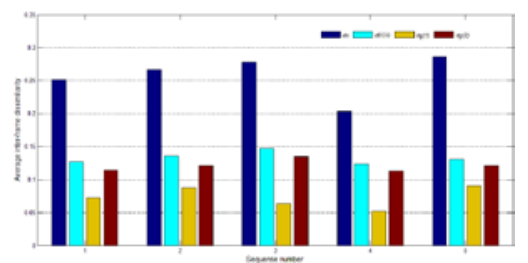

Fig. 12. Overall accuracy of three gating methods.

\section{Discussion}

For the ICUS sequences without recording of ECG signals, the heart rate is estimated according to the inter-frame dissimilarity based on the NCC of the average intensities [10]. However, such a global dissimilarity measure may result in the sensitivity of the estimation to image noises and non-cardiac dynamic factors. Meanwhile, the heart rate may not be regular during the catheter pullback. Therefore, the estimated average heart rate may not reflect real-time changes in the actual cardiac rate.

The pass-band center of the filter depends on the average heart rate. Because a higher variability in the heart rate may result in the occurrence of more distorted signals, the pass-band width should vary for different pullback sequences, instead of showing a constant value. For example, the heartbeat rhythm of a patient suffering from severe arrhythmia may be considerably unsteady. Therefore, the passband width should be larger than $0.6 R$ to include all frequencies contributing to periodic components of the signal and to avoid the removal of useful frequencies.

The threshold, $\varepsilon$, is the key to the selection of dynamic signals. A low $\varepsilon$ may lead to the selection of more motionless or interfered signals. However, a too-high $\varepsilon$ may result in the selected signals not completely reflecting the cardiac cycles. Although we set it as 3 and have obtained satisfactory gating results in our experiments with 31 in vivo pullback datasets, we have not found an intelligent scheme to automatically determine the $\varepsilon$ that is suitable for different ICUS pullback sequences. Further research on this topic will be performed.

\section{Conclusions}

A fully automated method that detects a 1-D phase signal reflecting the cardiac cycles from a continuous pullback ICUS image sequence is presented. The method is based on the quasi-periodic evolution of local image intensities along the sequence. The impact of image noises, non-vessel region, and useless textures on the detected signal is effectively reduced by discarding components produced by non-cardiac motion factors and the irregular heart rate. Neither a training phase nor prior 
information about the underlying anatomy is required. Moreover, the routinely acquired gray scale ICUS images are directly input without any high level preprocessing procedures. According to the quantitative comparison experiments with in vivo data, FIR filter is more suitable than IIR for filtering a 1-D signal correlating with cardiac cycles. The gating accuracy does not improve with increasing filter order. Furthermore, the gating results are not sensitive to FIR windows. Concerning the passband width, we plan to determine its inherent relationship with heart rate and intelligently select it for different cases, especially cases that present a significantly irregular cardiac profile.

\section{Acknowledgments}

This work was supported by National Nature Science Foundations of China (No. 61372042) and the Fundamental Research Funds for the Central Universities (No. 2014ZD31).

\section{References}

[1] D. Dutta, A.M. Mahmoud, S.A. Leers, et al., Motion artifact reduction in ultrasound based thermal strain imaging of atherosclerotic plaques using time series analysis, IEEE Transactions on Ultrasonics, Ferroelectrics, and Frequency Control 60 (2013), 1660-1668.

[2] Z. Sun and J. Wang, Compensation of in-plane rigid motion for in vivo intracoronary ultrasound image sequence, Computers in Biology and Medicine 43 (2013), 1077-1085.

[3] D. Guo and P. Richardson, Detection of cardiac cycle from intracoronary ultrasound, Ultrasound in Medicine \& Biology 32 (2006), 345-356.

[4] S.K. Nadkarni, D.R. Boughner and A. Fenster, Image-based cardiac gating for three-dimensional intravascular ultrasound, Ultrasound in Medicine \& Biology 31 (2005), 53-63.

[5] H. Zhu, K.D. Oakeso and M.H. Friedman, Retrieval of cardiac phase from IVUS sequences, Proceedings of SPIE Conference on Medical Imaging 2003: Ultrasonic Imaging and Signal Processing 5035 (2003),135-146.

[6] M. Rosales, P. Radeva, O. Rodriguez-Leor, et al., Modeling of image-catheter motion for 3-D IVUS, Medical Image Analysis 13 (2009), 91-104.

[7] J. Barajas, K.L. Caballero, O. Rodriguez-Leor and P. Radeva, Cardiac phase extraction in IVUS sequences using 1-D gabor filters, Proceedings of 29th Annual International Conference of the IEEE Engineering in Medicine and Biology Society (EMBS 2007), Los Alamitos, Aug 22-26, 2007, pp. 343-346.

[8] M.M.S. Matsumoto, T. Yoneyama, P.A. Lemos, et al., Cardiac phase detection in intravascular ultrasound images, Proceedings of SPIE Conference on Medical Imaging, San Diego, CA, USA, 6920 (2008), 69200D-69200D-9.

[9] S.A. de Winter, R. Hamers, M. Degertekin, et al., Retrospective image-based gating of intracoronary ultrasound images for improved quantitative analysis: the Intelligate method, Catheterization and Cardiovascular Interventions 61 (2004), 84-94.

[10] S.M. O'Malley, J.F. Granada, S. Carlier, et al., Image-based gating of intravascular ultrasound pullback sequences, IEEE Transactions on Information Technology in Biomedicine 12 (2008), 299-306.

[11] Z. Sun and Q. Yan, An off-line gating method for suppressing motion artifacts in ICUS sequence, Computers in Biology and Medicine 40 (2010), 860-868.

[12] C. Gatta, O. Pujol, R. Leor, et al., Robust image based IVUS pullbacks gating, Proceedings of 11th International Conference on Medical Image Computing and Computer-Assisted Intervention (MICCAI 2008), Heidelberg, Springer, 5242 (2008), 518-525.

[13] C. Gatta, S. Balocco, F. Ciompi, et al., Real-time gating of IVUS sequences based on motion blur analysis: method and quantitative validation, Proceedings of 13th International Conference on Medical Image Computing and ComputerAssisted Intervention (MICCAI 2010), Heidelberg, Springer, 2010, pp. 59-67.

[14] A. Hernàndez-Sabaté, D. Gil, J. Garcia-Barnés, et al., Image-based cardiac phase retrieval in intravascular ultrasound sequences, IEEE Transactions on Ultrasonics, Ferroelectrics, and Frequency Control 58 (2011), 60-72.

[15] H. Sundar, A. Khamene, L. Yatziv, et al., Automatic image-based cardiac and respiratory cycle synchronization and gating of image sequences, Proceedings of 12th International Conference on Medical Image Computing and ComputerAssisted Intervention (MICCAI 2009), 3rd ed. Heidelberg, Springer, 2009, pp. 381-388. 
[16] G.G. Isguder, G. Unal, M. Groher, et al., Manifold learning for image-based gating of intravascular ultrasound (IVUS) pullback sequences, Proceedings of 5th International Workshop on Medical Imaging and Augmented Reality (MIAR 2010) Beijing,China; Sep 19-20 2010, 6326 (2010), 139-148.

[17] C. Wachinger, M. Yigitsoy, E.J. Rijkhorst, et al., Manifold learning for image-based breathing gating in ultrasound and MRI, Medical Image Analysis 16 (2012), 806-818.

[18] S.K. Nadkarni, H. Austin, G. Mills, et al., A pulsating coronary vessel phantom for two- and three-dimensional intravascular ultrasound studies, Proceedings of 4th International Conference on Medical Image Computing and Computer-Assisted Intervention (MICCAI 2001), 2208 (2001), 1164-1165. 